\title{
Freqüência de síndrome metabólica em crianças e adolescentes com sobrepeso e obesidade
}

\author{
Frequency of metabolic syndrome in overweight and obese children and adolescents
}

\author{
Caroline de Gouveia Buff', Eliete Ramos ${ }^{1}$, Fabíola Isabel S. Souza ${ }^{2}$, Roseli Oselka S. Sarni
}

\section{RESUMO}

Objetivo: Avaliar a freqüência de síndrome metabólica em crianças e adolescentes com sobrepeso ou obesos, além de relacionar a sua presença com variáveis socioeconômicas, atividade física e risco cardiovascular familiar.

Métodos: Por meio de estudo transversal, 59 pacientes com sobrepeso/obesidade, matriculados em ambulatório universitário, foram avaliados entre janeiro de 2004 e junho de 2006 . Coletaramse os seguintes dados: nível socioeconômico, escolaridade materna, risco cardiovascular familiar e atividade física. Utilizou-se o percentil do índice de massa corpórea (IMC) para classificar a condição nutricional. Considerou-se síndrome metabólica quando presentes três ou mais das seguintes alterações: triglicérides $(\geq 110 \mathrm{mg} / \mathrm{dL})$, HDL-c ( $\leq 40 \mathrm{mg} / \mathrm{dL})$, circunferência abdominal ( $\geq$ percentil 90$)$, resistência insulínica (glicemia $>100 \mathrm{mg} / \mathrm{dL}$ ) e pressão arterial ( $\geq$ percentil 90). A análise estatística foi descritiva.

Resultados: Dos pacientes, 52,5\% eram do gênero masculino e a média de idade foi 10,9 $\pm 0,5$ anos. A síndrome metabólica esteve presente em $42,4 \%$. Inadequações das variáveis que compõem a síndrome metabólica foram observadas em $88,1 \%$ para a circunferência abdominal; $47,5 \%$ com hipertensão arterial; 23,7\% com resistência insulínica; 42,4\% com aumento dos triglicérides e 6,8\% com elevação do HDL-c. Não se observou associação estatisticamente significante entre síndrome metabólica e gênero, idade, desenvolvimento puberal, renda per capita, escolaridade materna, horas de televisão/dia, horas de atividade física ou risco cardiovascular familiar.

Conclusões: É alta a prevalência de síndrome metabólica em crianças e adolescentes obesos avaliados e, dentre os seus componentes, a circunferência abdominal e a pressão arterial elevadas são as mais freqüentes.

Palavras-chave: obesidade; resistência à insulina; criança; adolescente; circunferência abdominal.

\section{ABSTRACT}

Objective: Evaluate metabolic syndrome in overweight/ obese children and adolescents and to study the association between this syndrome and socioeconomic variables, physical activity and familiar cardiovascular risk.

Methods: This cross-sectional study enrolled 59 children and adolescents with overweight/obesity in an outpatient university clinic, from January 2004 to June 2006. The following variables were studied: socioeconomic level, maternal schooling, familiar cardiovascular risk and physical activity. Body mass index (BMI) percentile was used to classify the nutritional status. Metabolic syndrome was considered when three or more of the following were present: triglycerides $\geq 110 \mathrm{mg} / \mathrm{dL}$ or HDL-c $\leq 40 \mathrm{mg} / \mathrm{dL}$, waist circumference $\geq 90^{\text {th }}$ percentile, insulin resistance (glucose level $>100 \mathrm{mg} / \mathrm{dL}$ ) and blood pressure $\mathrm{BP} \geq 90^{\text {th }}$ percentile. Descriptive statistical analysis was applied.

Results: Out of the 59 children, mean age was $10.9 \pm 0.48$ years and $52.5 \%$ were male. Metabolic syndrome was present in $42.4 \%$. Altered components of the metabolic syndrome were: abdominal circumference in $88.1 \%$, blood pressure $47.5 \%$, insulin resistance $23.7 \%$, triglycerides $42.4 \%$ and HDL-c $6.8 \%$. No significant association was found between the presence of metabolic syndrome and gender, age, pubertal development, per capita income, maternal schooling, daily hours of TV viewing, weekly hours of physical activity $(p=0.942)$ or familiar cardiovascular risk.

Conclusions: The prevalence of metabolic syndrome is high among the evaluated overweight/obese children and adolescents. Altered abdominal circumference and high blood pressure were the most frequent components of the syndrome.

Key-words: obesity; insulin resistance; child; adolescent; abdominal circumference.

Endereço para correspondência

Roseli Oselka S. Sarni

Rua René Zamlutti, 94, apto. 92 - Vila Mariana

CEP 04116-260 - São Paulo/SP

E-mail: rssarni@uol.com.br

Recebido em: 16/3/2007

Aprovado em: 30/7/2007 


\section{Introdução}

A obesidade é uma doença crônica que cresce de forma epidêmica em todo o mundo, atingindo todas as faixas etárias. Tem etiologia multifatorial, associando-se a fatores genéticos, ambientais e comportamentais, e preocupa devido às suas repercussões a curto e longo prazo ${ }^{(1)}$.

Em crianças e adolescentes americanos, observa-se que a prevalência de obesidade triplicou nas últimas três décadas. Nos Estados Unidos, cerca de 15\% das crianças e adolescentes são obesos, considerando-se como critério para diagnóstico o índice de massa corpórea (IMC) acima do percentil 95 para idade e gênero ${ }^{(2)}$. Estudo populacional recente realizado na cidade de Santos, São Paulo, envolvendo 10.882 crianças com idade entre sete e dez anos, revelou prevalência de sobrepeso e obesidade de 15,7 e $18 \%$, respectivamente ${ }^{(3)}$.

A obesidade na infância associa-se à sua persistência na vida adulta e também a uma série de co-morbidades como dislipidemia, hipertensão arterial sistêmica, problemas psicossociais, alterações do metabolismo da glicose, problemas ortopédicos, apnéia do sono, síndrome dos ovários policísticos e esteatose hepática ${ }^{(4)}$.

Destaca-se atualmente, como foco de estudo, a síndrome metabólica, reconhecido fator de risco para o desenvolvimento de doença cardiovascular e fortemente associada ao excesso de peso. É bem descrita em adultos e caracteriza-se pela presença de três ou mais dos seguintes critérios: pressão arterial elevada, HDL colesterol baixo, triglicérides elevados, presença de resistência insulínica e obesidade abdominal ${ }^{(5)}$. Dados recentes estimam que a síndrome metabólica afeta $22 \%$ da população adulta norte-americana, incluindo $7 \%$ de homens e $6 \%$ de mulheres entre 20 e 29 anos, faixa etária próxima da adolescência ${ }^{(6)}$.

Há poucos estudos avaliando a prevalência de síndrome metabólica em crianças e adolescentes, não existindo consenso sobre critérios e pontos de corte a serem utilizados, os quais variam de estudo para estudo ${ }^{(7-9)}$. Entre os trabalhos publicados, verificam-se freqüências de $23,3 \%$ em crianças portuguesas obesas ${ }^{(7)}, 28,7 \%$ em adolescentes (12 a 18 anos) americanos com obesidade ${ }^{(8)}$ e $17,3 \%$ em crianças obesas com sete a dez anos, no Distrito Federal, Brasil ${ }^{(9)}$.

Assim, diante da importância da obesidade e de suas repercussões a curto e longo prazo, realizou-se este estudo, cujo objetivo foi avaliar a freqüência de síndrome metabólica em crianças e adolescentes com sobrepeso/obesidade matriculados em ambulatório de serviço universitário de referência, além de relacionar a presença da síndrome metabólica com variáveis socioeconômicas, risco cardiovascular familiar e atividade física.

\section{Método}

Por meio de estudo transversal, foram avaliados 59 pacientes com sobrepeso e obesidade matriculados no Ambulatório de Obesidade do Serviço de Crescimento, Desenvolvimento e Terapia Nutricional do Departamento de Pediatria da Faculdade de Medicina do ABC (FMABC), Santo André, São Paulo. Foram incluídos no estudo todos os pacientes que ingressaram no serviço entre janeiro de 2004 e junho de 2006. Foram excluídos os portadores de doenças crônicas como: nefropatias, endocrinopatias, hepatopatias, doenças reumatológicas e baixa estatura (escore $Z$ de estatura/idade $<-2)$. A pesquisa foi aprovada pelo Comitê de Ética em Pesquisa da FMABC.

A avaliação socioeconômica foi analisada a partir das variáveis "salário mínimo per capita" (SMPC) e "escolaridade materna”. Estabeleceu-se como baixo nível socioeconômico o SMPC inferior a $0,5^{(10)}$ e a baixa escolaridade materna quando inferior a quatro anos.

Os antecedentes de risco familiar para doença cardiovascular foram considerados presentes quando relatado que pais, irmãos, avós, tios e tias tivessem apresentado obesidade, hipertensão, dislipidemia, diabetes, tabagismo e/ou doenças cardiovasculares, antes dos 55 anos para homens e dos 65 anos para mulheres ${ }^{(11)}$

A presença de atividades diárias foi avaliada segundo a prática de atividade física regular extracurricular (número de vezes na semana e duração da atividade), nível de sedentarismo (horas em televisão, vídeo game e computador por dia) e horas de sono noturno.

Para a avaliar a condição nutricional, foram obtidos os dados de peso e estatura ${ }^{(12)}$. As crianças e adolescentes foram classificadas de acordo com o percentil do IMC em eutróficos (IMC $<$ P85), sobrepeso (P85 $\leq \mathrm{IMC}<\mathrm{P} 95$ ) e obesos $(\mathrm{IMC} \geq \mathrm{P} 95)^{(13)}$. Utilizou-se também o índice de estatura para idade sob a forma do escore $Z$.

O estadiamento puberal foi avaliado por um único pediatra e classificado de acordo com a presença de caracteres sexuais secundários em ambos os gêneros $(\mathrm{M}=$ mamas e $\mathrm{G}=$ testículos), como proposto por Marshall \& Tanner ${ }^{(14)}$.

A circunferência abdominal foi aferida por meio de fita inextensível, graduada em milímetros, no ponto médio 
entre a décima costela e a crista ilíaca superior. Foram considerados inadequados os valores de circunferência abdominal iguais ou maiores ao percentil 90 para gênero e idade ${ }^{(15)}$.

Finalmente, a aferição da pressão arterial foi obtida segundo preconização do Task Force de 2004 e classificada segundo gênero, idade e estatura, sendo o ponto de corte pressão arterial sistólica ou diastólica igual ou superior ao percentil $90^{(16)}$.

Quanto aos exames laboratoriais, após jejum de 12 horas, coletou-se $10 \mathrm{~mL}$ de sangue por venopunção periférica para a dosagem do perfil lipídico (LDL-c, HDL-c e triglicérides), no período da manhã. Adotou-se como ponto de corte o critério de Kwiterovich $^{(17)}$. Para a glicemia, estabeleceu-se como ponto de corte valor superior a $100 \mathrm{mg} / \mathrm{dL}^{(18)}$.

Para caracterizar a síndrome metabólica, utilizou-se o critério proposto por Cook et a ${ }^{(8)}$ modificado, que considera a sua presença diante de três ou mais das seguintes alterações: triglicérides $\geq 110 \mathrm{mg} / \mathrm{dL}$, HDL-c $\leq 40 \mathrm{mg} / \mathrm{dL}$, circunferência abdominal $\geq \mathrm{P} 90$, resistência insulínica (glicemia $>100 \mathrm{mg} / \mathrm{dL}$ ) e pressão arterial sistólica ou diastólica $\geq \mathrm{P} 90$.

$\mathrm{Na}$ análise estatística, utilizou-se o SPSS 13.0 e o EpiInfo 2000. Foram construídas tabelas de freqüência e aplicado o teste do qui-quadrado para comparar as variáveis de forma qualitativa e o cálculo da Odds Ratio (OR) para verificar a associação entre as variáveis estudadas, considerando-se $p<0,05$ como significante.

\section{Resultados}

Dos pacientes que compuseram o estudo, 31/59 (52,5\%) eram do gênero masculino, a média de idade foi 10,9 90,48 anos, e 29/59 (49,1\%) apresentavam desenvolvimento dos caracteres sexuais secundários (estadios 2, 3, 4 ou 5), conforme Tabela 1. Em relação à condição nutricional, 52/59 $(88,1 \%)$ e $7 / 59(11,8 \%)$ tinham obesidade e sobrepeso, respectivamente. Dentre as crianças e os adolescentes avaliados, apresentavam renda per capita inferior a meio salário mínimo 28/52 (53,8\%) e mãe com baixa escolaridade (inferior a quatro anos) 8/59 (13,6\%), de acordo com a Tabela 1.

Quanto às atividades diárias, 27/59 (45,8\%), 42/59 $(71,2 \%)$ e $31 / 59(52,5 \%)$ pacientes estudados assistiam mais do que quatro horas de televisão por dia, dormiam menos do que dez horas por noite e praticavam menos de duas horas de atividade física leve por semana, respectivamente.

A síndrome metabólica esteve presente em 25/59 $(42,4 \%)$ dos pacientes avaliados. Inadequações das variáveis
Tabela 1 - Caracterização da população estudada

\begin{tabular}{|c|c|c|c|}
\hline Variável & & $\mathbf{N}$ & $\%$ \\
\hline Gênero & $\begin{array}{c}\text { Masculino } \\
\text { Feminino }\end{array}$ & $\begin{array}{l}31 \\
28\end{array}$ & $\begin{array}{l}52,5 \% \\
47,5 \%\end{array}$ \\
\hline Idade & $\begin{array}{c}2-7 \text { anos } \\
7-10 \text { anos } \\
>10 \text { anos }\end{array}$ & $\begin{array}{c}7 \\
18 \\
34\end{array}$ & $\begin{array}{l}11,9 \% \\
30,5 \% \\
57,6 \%\end{array}$ \\
\hline Estadio de Tanner & $\begin{array}{l}\text { M1 ou G1 } \\
\text { M2 ou G2 } \\
\text { M3 ou G3 } \\
\text { M4 ou G4 } \\
\text { M5 ou G5 }\end{array}$ & $\begin{array}{c}30 \\
10 \\
5 \\
13 \\
1\end{array}$ & $\begin{array}{c}50,8 \% \\
16,9 \% \\
8,5 \% \\
22,0 \% \\
1,7 \%\end{array}$ \\
\hline Condição nutricional & $\begin{array}{c}\mathrm{P} 85 \leq \mathrm{IMC}<\mathrm{P} 95 \\
\geq \mathrm{P} 95\end{array}$ & $\begin{array}{c}7 \\
52\end{array}$ & $\begin{array}{l}11,9 \% \\
88,1 \%\end{array}$ \\
\hline Renda per capita & $\begin{array}{l}<0,5 S M \\
>0,5 S M\end{array}$ & $\begin{array}{l}28 \\
24\end{array}$ & $\begin{array}{l}53,8 \% \\
46,2 \%\end{array}$ \\
\hline Escolaridade materna & $\begin{array}{c}<4 \text { anos } \\
4-8 \text { anos } \\
>8 \text { anos }\end{array}$ & $\begin{array}{c}8 \\
18 \\
25\end{array}$ & $\begin{array}{l}13,6 \% \\
30,5 \% \\
42,4 \%\end{array}$ \\
\hline Televisão & $\begin{array}{l}<4 \mathrm{~h} / \mathrm{dia} \\
\geq 4 \mathrm{~h} / \mathrm{dia}\end{array}$ & $\begin{array}{l}32 \\
27\end{array}$ & $\begin{array}{l}54,2 \% \\
45,8 \%\end{array}$ \\
\hline Sono noturno & $\begin{array}{l}\geq 10 \text { horas } \\
<10 \text { horas }\end{array}$ & $\begin{array}{l}17 \\
42\end{array}$ & $\begin{array}{l}28,8 \% \\
71,2 \%\end{array}$ \\
\hline Atividade física & $\begin{array}{l}\geq 2 \mathrm{~h} / \mathrm{sem} \\
<2 \mathrm{~h} / \mathrm{sem}\end{array}$ & $\begin{array}{l}28 \\
31\end{array}$ & $\begin{array}{l}47,5 \% \\
52,5 \%\end{array}$ \\
\hline Circunferência abdominal & $\begin{array}{l}<\mathrm{P} 90 \\
\geq \mathrm{P} 90\end{array}$ & $\begin{array}{c}52 \\
7\end{array}$ & $\begin{array}{l}88,1 \% \\
11,9 \%\end{array}$ \\
\hline Pressão arterial sistólica & $\begin{array}{l}<\mathrm{P} 90 \\
\geq \mathrm{P} 90\end{array}$ & $\begin{array}{l}38 \\
19\end{array}$ & $\begin{array}{l}64,4 \% \\
32,2 \%\end{array}$ \\
\hline Pressão arterial diastólica & $\begin{array}{l}<\mathrm{P} 90 \\
\geq \mathrm{P} 90\end{array}$ & $\begin{array}{l}32 \\
25\end{array}$ & $\begin{array}{l}54,2 \% \\
42,4 \%\end{array}$ \\
\hline HDL-C & $\begin{array}{l}\text { Adequado } \\
\text { Inadequado }\end{array}$ & $\begin{array}{c}55 \\
4\end{array}$ & $\begin{array}{c}93,2 \% \\
6,8 \%\end{array}$ \\
\hline LDL-C & $\begin{array}{l}\text { Adequado } \\
\text { Inadequado }\end{array}$ & $\begin{array}{l}47 \\
12\end{array}$ & $\begin{array}{l}79,7 \% \\
20,3 \%\end{array}$ \\
\hline Triglicerídios & $\begin{array}{l}\text { Adequado } \\
\text { Inadequado }\end{array}$ & $\begin{array}{l}34 \\
25\end{array}$ & $\begin{array}{l}57,6 \% \\
42,4 \%\end{array}$ \\
\hline Glicemia & $\begin{array}{l}>100 \mathrm{mg} / \mathrm{dL} \\
\leq 100 \mathrm{mg} / \mathrm{dL}\end{array}$ & $\begin{array}{l}14 \\
45\end{array}$ & $\begin{array}{l}23,7 \% \\
76,3 \%\end{array}$ \\
\hline
\end{tabular}

SM - salário mínimo

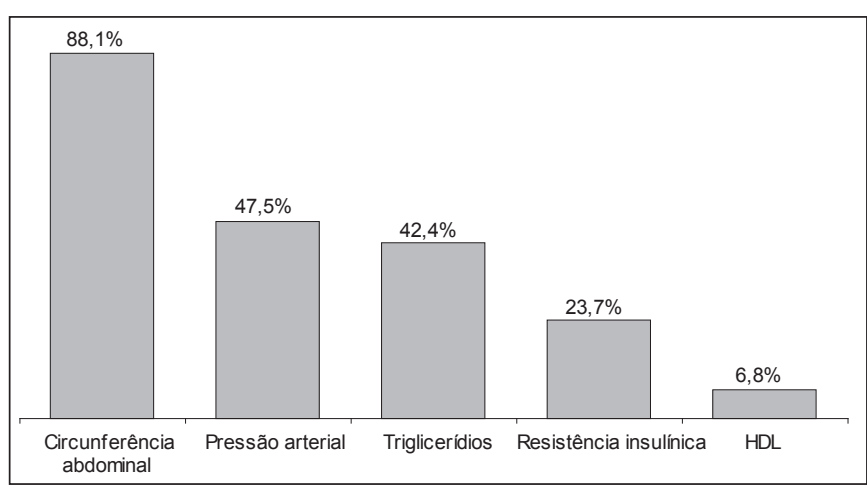

Gráfico 1 - Percentual de inadequação dos componentes da síndrome metabólica 
que compõem a síndrome metabólica foram observadas em: $52 / 59(88,1 \%)$ no que concerne à circunferência abdominal; $28 / 59(47,5 \%)$ em relação à hipertensão arterial $(32,2 \%$ sistólica e 42,4\% diastólica); 13/59 (23,7\%) na resistência insulínica; 25/59 (42,4\%) nos triglicérides e 4/59 (6,8\%) no HDL-c (Gráfico 1). Quanto ao LDL-c, encontrou-se $12 / 59(20,3 \%)$ de inadequação.
Não foi observada associação estatisticamente significante entre a presença de síndrome metabólica e gênero, idade, desenvolvimento puberal, renda per capita, escolaridade materna, horas de televisão diárias, horas de sono noturno, horas de atividade física, conforme Tabela 2. Também não se observou associação emtre a síndrome metabólica e antecedentes familiares para doenças cardiovasculares (Tabela 3).

Tabela 2 - Associação entre as variáveis estudadas e a presença de síndrome metabólica

\begin{tabular}{|c|c|c|c|c|c|}
\hline \multirow{4}{*}{ Variável } & & Com & Sem & \multirow{4}{*}{ OR (IC95\%) } & \multirow{4}{*}{ Valor de $p$} \\
\hline & & \multicolumn{2}{|c|}{ Síndrome metabólica } & & \\
\hline & & $n=25$ & $n=34$ & & \\
\hline & & N \% & $\mathbf{N} \%$ & & \\
\hline Gênero & Masculino & $14(56)$ & $17(46)$ & $1,3(0,4-4,1)$ & 0,648 \\
\hline Idade & $\geq 10$ anos & $14(56)$ & $20(59)$ & $0,9(0,3-2,9)$ & 0,828 \\
\hline Desenvolvimento puberal & Presente & $11(44)$ & $18(53)$ & $0,7(0,2-2,3)$ & 0,491 \\
\hline Renda per capita & $\leq 0,5 \mathrm{SM}$ & $11(44)$ & $17(50)$ & $1,1(0,3-3,9)$ & 0,895 \\
\hline Escolaridade materna & $<4$ anos & $5(8)$ & $3(8)$ & $3,1(0,5-19,6)$ & 0,141 \\
\hline Horas de televisão por dia & $\geq 4$ horas & $14(56)$ & $13(38)$ & $2,1(0,6-6,8)$ & 0,175 \\
\hline Horas de sono noturno & $\geq 10$ horas & $4(16)$ & $13(38)$ & $0,3(0,1-1,3)$ & 0,062 \\
\hline Horas atividade física & $<2 \mathrm{~h} / \mathrm{sem}$ & $12(48)$ & $16(47)$ & $1,0(0,3-3,2)$ & 0,942 \\
\hline LDL-C & Aumentado & $7(28)$ & $5(15)$ & $1,5(0,3-3,7)$ & 0,559 \\
\hline
\end{tabular}

SM - salário mínimo

Tabela 3 - Associação entre os antecedentes familiares e a síndrome metabólica

\begin{tabular}{|c|c|c|c|c|}
\hline \multirow{4}{*}{ Variável } & \multicolumn{2}{|c|}{ Síndrome metabólica } & \multirow{4}{*}{ OR (IC95\%) } & \multirow{4}{*}{ Valor de $p$} \\
\hline & \multirow{3}{*}{$\begin{array}{c}\text { Com } \\
n=25\end{array}$} & \multirow{2}{*}{$\begin{array}{l}\text { Sem } \\
n=34\end{array}$} & & \\
\hline & & & & \\
\hline & & N $\%$ & & \\
\hline Obesidade & $23(92)$ & $30(88)$ & $0,4(0,01-5,9)$ & 0,418 \\
\hline Hipertensão arterial & $20(80)$ & $29(85)$ & $0,7(0,1-3,2)$ & 0,422 \\
\hline Dislipidemias & $16(64)$ & $16(47)$ & $2,0(0,6-6,6)$ & 0,196 \\
\hline Evento cardiovascular precoce & $15(60)$ & $20(59)$ & $1,0(0,3-3,4)$ & 0,927 \\
\hline Diabetes melito & $15(60)$ & $18(53)$ & $1,3(0,4-4,3)$ & 0,589 \\
\hline
\end{tabular}




\section{Discussão}

Encontrou-se alto percentual de síndrome metabólica na população estudada (42,3\%). Essa freqüência é superior a outros estudos populacionais com crianças ${ }^{(7,9)}$ e adolescentes obesos $^{(8)}$. Possivelmente, esta alta frequência decorra da casuística ser proveniente de serviço universitário, que atende os casos mais graves de obesidade e, também, pela diversidade de critérios utilizados para caracterizar a síndrome em diferentes estudos. Chen e Berenson ${ }^{(19)}$, em publicação recente, ressaltaram a limitação dos critérios para caracterizar a síndrome metabólica na faixa etária pediátrica e reforçaram a importância de uma definição universal. Entretanto, não deixa de ser bastante preocupante o fato de quase $50 \%$ dos pacientes, ainda bastante jovens (média de idade de 10,9 anos), serem encaminhados ao serviço de referência com uma série de co-morbidades associadas.

Dentre os critérios diagnósticos para síndrome metabólica, a obesidade abdominal foi a que mostrou maior percentual de inadequação. $\mathrm{O}$ achado reforça a hipótese de alguns pesquisadores que a obesidade, por si só, seria um determinante expressivo da síndrome metabólica, mesmo durante a infância e a adolescência ${ }^{(20)}$. A presença da adiposidade central é fator de risco comprovado para o desenvolvimento de dislipidemias e resistência insulínica na faixa etária pediátrica ${ }^{(15)}$.

A relação entre obesidade, síndrome metabólica e doença aterosclerótica é bem conhecida em adultos ${ }^{(5)}$. Embora crianças e adolescentes não apresentem doença aterosclerótica franca, as crianças obesas mostram um perfil de risco cardiovascular compatível com seu desenvolvimento precoce, isto é, alterações de perfil lipídico, da pressão arterial e da glicemia $^{(21-24)}$. Estudo de coorte em indivíduos jovens mostra que a presença de síndrome metabólica está associada a espessamento endotelial avaliado por ultra-sonografia com medida de fluxo de carótida, sugerindo que o processo tenha início já na infância ${ }^{(25)}$. Entretanto, há limitações nos estudos longitudinais disponíveis, não sendo possível mensurar a influência dessas morbidades associadas no desenvolvimento de doenças futuras, especialmente diabetes melito e doença aterosclerótica $^{(21,23)}$.
Houve predominância do baixo nível econômico nas famílias dos pacientes avaliados. É comum a associação entre nível econômico desfavorável e aparecimento de distúrbios nutricionais, incluindo a obesidade, com risco aumentado para o desenvolvimento de co-morbidades ${ }^{(24)}$. Em populações de baixo nível socioeconômico, a obesidade co-existe com outras carências nutricionais, destacando-se a carência de micronutrientes, entre eles os antioxidantes (vitamina A, vitamina E, selênio, zinco, cobre), o que colabora para exacerbar o estresse oxidativo e elevar o risco de doenças crônicas não-transmissíveis ${ }^{(26)}$.

A maioria dos pacientes estudados, independentemente da presença da síndrome metabólica, mostrou estilo de vida sedentário, pouca atividade física regular e tempo excessivo gasto com televisão, videogame e computador. Sabe-se que a inatividade física é um dos fatores mais importantes para o desenvolvimento de doenças crônicas ${ }^{(27)}$ e, quando presente na infância, tende a persistir na vida adulta.

Contrariamente a outros estudos da literatura, não se observou associação entre a síndrome metabólica e a história familiar para doença cardiovascular ${ }^{(28,29)}$. A falta de associação pode derivar do desconhecimento da família em relação aos antecedentes pesquisados ou, mesmo, decorrer da não realização rotineira de exames para identificar de forma precoce e, consequentemente, prevenir de modo efetivo as doenças cardiovasculares.

O nível socioeconômico, as atividades de vida diária e o risco cardiovascular familiar não mostraram associação com a presença da síndrome metabólica. Cabe ressaltar que há limitação no número de pacientes avaliados, o que pode ter contribuído para a falta de associação entre a síndrome metabólica e as variáveis estudadas.

Os resultados obtidos permitem concluir que é alta a prevalência de síndrome metabólica entre crianças e adolescentes com sobrepeso/obesidade avaliados e, dentre os seus componentes, a circunferência abdominal e a pressão arterial elevadas são os mais alterados. Tais alterações podem contribuir para o desenvolvimento precoce de doenças crônicas, o que remete à importância da prevenção, estimulando hábitos alimentares e estilo de vida saudáveis. 


\section{Referências bibliográficas}

1. Flegal KM, Carroll MID, Johnson CL. Prevalence and trends in obesity among US adults 1999-2000. JAMA 2002;288:1723-7.

2. Ogden CL, Flegal KM, Carroll MID, Johnson CL. Prevalence and trends in obesity among US children and adolescents 1999-2000. JAMA 2002;288:1728-32.

3. Costa RF, Cintra IP, Fisberg M. Prevalência de sobrepeso e obesidade em escolares da cidade de Santos. Arq Bras Endocrinol Metab 2006;50:60-7.

4. World Health Organization. Obesity: preventing and managing the global epidemic: report of a WHO Consultation. WHO Technical Report Series 894. Geneva: World Health Organization; 2000.

5. National Institute of Health: Adult treatment panel III. Washington: US Government Print Office, 2001.

6. Ford ES. Prevalence of the metabolic syndrome among US adults: findings from the third National Health and Nutrition Examination Survey. JAMA 2002;287:356-9.

7. Antunes $H$, Resende D, Paiva M, Santos C. Metabolic Syndrome in a paediatric obese population by the IDF new criteria. J Pediatric Gastroenterol Nutr 2006;42:9-10.

8. Cook S, Weitzman M, Auinger P, Nguyen M, Dietz WH. Prevalence of a metabolic syndrome phenotype in adolescents. Arch Pediatrics Adolesc Med 2003;157:821-7.

9. Ferreira AP, Oliveira CE, Franca NM. Metabolic syndrome and risk factors for cardiovascular disease in obese children: the relationship with insulin resistance (HOMA-IR). J Pediatr (Rio J) 2007;83:21-6.

10. Pedrazzani ES, Mello DA, Pripas S, Fucci M, Barbosa CA, Santoro MC. Intestinal helminthiasis. II. Prevalence and correlation with income, family size, anemia and nutritional status. Rev Saude Publica 1988;22:384-9.

11. Kavey RE, Daniels SR, Lauer RM, Atkins DL, Hayman LL, Taubert K. American Heart Association. American Heart Association guidelines for primary prevention of atherosclerotic cardiovascular disease beginning in childhood. Circulation 2003;107:1562-6.

12. World Health Organization. Physical Status: the use and interpretation of anthropometry. WHO Technical Report Series 854. Geneva: World Health Organization; 1995.

13. Kuczmarski RJ, Ogden CL, Grummer-Strawn LM, Flegal KM, Guo SS, Wei R et al. CDC growth charts: United States. Adv Data 2000;8:1-7

14. Marshall WA, Tanner JM. Variations in pattern of pubertal changes in girls. Arch Dis Child 1969;44:291-303.

15. Freedman DS, Serdula MK, Srinivasan SR, Berenson GS. Relation of circumferences and skinfold thicknesses to lipid and insulin concentrations in children and adolescents: the Bogalusa Heart Study. Am J Clin Nutr 1999;69:308-17.
16. National High Blood Pressure Education Program Working Group on High Blood Pressure in Children and Adolescents. The fourth report on the diagnosis, evaluation, and treatment of high blood pressure in children and adolescents. Pediatrics 2004;114:555-76.

17. Kwiterovich PO. Triglyceride problems. In: Kwiterovich PO. Beyond cholesterol: the Johns Hopkins complete guide for avoiding heart disease. Baltimore: Johns Hopkins University Press; 1989. p. 122-42.

18. American Diabetes Association. Diagnosis and classification of diabetes mellitus. Diabetes Care 2005;28:S37-42.

19. Chen W, Berenson GS. Metabolic syndrome: definition and prevalence in children. J Pediatr (Rio J) 2007;83:1-2.

20. Srinivasan SR, Myers L, Berenson GS. Predictability of childhood adiposity and insulin for developing insulin resistance syndrome (syndrome $\mathrm{X}$ ) in young adulthood: the Bogalusa Heart Study. Diabetes 2002;51:204-9.

21. Sinaiko A. Obesity, insulin resistance and the metabolic syndrome. J Pediatr (Rio J) 2007;83:3-4.

22. Chen W, Srinivasan SR, Li S, Xu J, Berenson GS. Metabolic syndrome variables at low levels in childhood are beneficially associated with adulthood cardiovascular risk: the Bogalusa Heart Study. Diabetes Care 2005;28:126-31.

23. Berenson GS, Srinivasan SR, Bao W, Newman WP, Tracy RE, Wattigney WA. Association between multiple cardiovascular risk factors and atherosclerosis in children and young adults. The Bogalusa Heart Study. N Engl J Med 1998;338:1650-6.

24. Kivimaki M, Lawlor DA, Smith GD, Keltikangas-Jarvinen L, Elovainio M, Vahtera $\mathrm{J}$ et al. Early socioeconomic position and blood pressure in childhood and adulthood: the Cardiovascular Risk in Young Finns Study. Hypertension 2006;47:39-44.

25. Tzou WS, Douglas PS, Srinivasan SR, Bond MG, Tang R, Chen W et al. Increased subclinical atherosclerosis in young adults with metabolic syndrome: the Bogalusa Heart Study. J Am Coll Cardiol 2005;46:457-63.

26. Sarni RO, Souza FI, Ramalho RA, Schoeps D, Kochi C, Catherino P et al Serum retinol and total carotene concentrations in obese pre-school children. Med Sci Monit 2005;11:510-4.

27. Marshall SJ, Biddle SJ, Gorely T, Cameron N, Murdey I. Relationships between media use, body fatness and physical activity in children and youth: a metaanalysis. Int J Obes Relat Metab Disord. 2004;28:1238-46.

28. Forti N, Diament J, Cardoso AL, Romaldini CC, Issler H. Fatores de risco para aterosclerose em crianças e adolescentes com história familiar de doença arterial coronariana prematura J Pediatr 2004;80:135-40.

29. Pankow JS, Jacobs DR, Steinberger J, Moran A, Sinaiko AR. Insulin resistance and cardiovascular disease risk factors in children of parents with the insulin resistance (metabolic) syndrome. Diabetes Care 2004;27:775-80. 